\title{
ARTICLE
}

Molecular Diagnostics

\section{Carbonic anhydrase 9 (CA9) expression in non-small-cell lung cancer: correlation with regulatory FOXP3+T-cell tumour stroma infiltration}

\author{
Alexandra Giatromanolaki ${ }^{1}$, Adrian L. Harris $\mathbb{D}^{2}$, Alison H. Banham ${ }^{3}$, Constantinos A. Contrafouris ${ }^{4}$ and Michael I. Koukourakis ${ }^{5}$
}

BACKGROUND: Low pH suppresses the proliferation and cytotoxic activity of CD8+ cytotoxic and natural killer lymphocytes. The hypoxia-regulated transmembrane protein, carbonic anhydrase CA9, converts carbon dioxide produced by the Krebs cycle to bicarbonate and protons that acidify the extracellular milieu. We examined whether CA9 is also involved in intratumoural immunosuppression pathways.

METHODS: A series of 98 tissue samples of primary non-small-cell lung carcinomas (NSCLC) from patients treated with surgery were analysed for the expression of CA9 and programmed-death ligand PD-L1 by cancer cells, and of FOXP3 by tumour-infiltrating lymphocytes (TILs).

RESULTS: There was no direct association of CA9 with PD-L1 expression or the density of TILs in the tumour stroma, but CA9 was directly related to the extent of FOXP3 + TIL density $(p=0.008)$. Double-stratification survival analysis showed that patients with high CA9 expression and low TIL score had significantly poorer survival compared with all other groups $(p<0.04)$. In a multivariate analysis stage ( $p<0.0001, \mathrm{HR} 1.95,95 \% \mathrm{Cl}$ : 1.3-2.7), TIL score $(p=0.05, \mathrm{HR} 0.55,95 \% \mathrm{Cl}: 0.2-1.0)$ was an independent prognostic variable of death events. CA9 expression by cancer cells is associated significantly with FOXP3+ regulatory T-cell abundance in the tumour stroma of NSCLC.

CONCLUSION: The study provides a basis for testing CA9 as a marker of resistance to immune-checkpoint inhibitors and as a therapeutic target to enhance the efficacy of immunotherapy.

British Journal of Cancer (2020) 122:1205-1210; https://doi.org/10.1038/s41416-020-0756-3

\section{BACKGROUND}

Immunotherapy with immune-checkpoint inhibitors has revolutionised the therapy of metastatic disease for several human malignancies, including non-small-cell lung cancer (NSCLC). AntiPD-1/PD-L1 monoclonal antibodies, alone or in combination with chemotherapy, have been approved for the treatment of advanced NSCLC, following randomised trials that confirmed the improvement of the overall survival and a better toxicity profile compared with chemotherapy. ${ }^{1}$ Nevertheless, despite the documented prolongation of life, the response rates to immunotherapy are less than $25 \%$ in previously untreated patients, which increases to $35 \%$ when tumours extensively express PD-L1. ${ }^{2}$ Moreover, responders will eventually relapse. Lack of PD-L1 expression, co-expression of other disrupting immunecheckpoint co-inhibitory molecules or impaired antigen presentation machinery by cancer cells contributes to the failure of antiPD-L1 therapy to control the disease. ${ }^{3}$

The tumour microenvironment is also strongly implicated in tumour evasion from immune surveillance. Intratumoural hypoxia, through HIF1a activation, promotes the secretion of cytokines (e.g. IL-6 and IL-8) involved in the differentiation of myeloid dendritic cells towards an immunosuppressive phenotype. ${ }^{4}$ Hypoxia also promotes HIF1a-dependent overexpression of CD47 or PD-L1 immune-checkpoint inhibitory molecules by cancer cells or tumour-infiltrating immune cells., ${ }^{5,6}$ Enhanced production of lactate, through anaerobic glycolysis and subsequent extracellular transport of lactate and protons by monocarboxylate transporters, is also a major pathway inducing acidosis in the tumour microenvironment. ${ }^{7}$ Another pathway that promotes intratumoural acidosis involves glutamine metabolism that feeds the Krebs cycle with glutamate and a-ketoglutarate, sustaining oxidative metabolism under hypoxia, producing $\mathrm{CO}_{2} \cdot{ }^{8} \mathrm{CO}_{2}$ is hydrated by the transmembrane protein carbonic anhydrase 9 (CA9) and converted to bicarbonate and protons that acidify the extracellular milieu. $^{9}$ In addition, hypoxia-induced bicarbonate transporters import $\mathrm{HCO}^{-}$ions, which combine with intracellular acid to produce $\mathrm{CO}_{2}$ that diffuses out of the cell, a gradient maintained by CA9. ${ }^{10}$ Low $\mathrm{pH}$ markedly suppresses the proliferation and cytotoxic activity of CD8+ cytotoxic and natural killer lymphocytes. ${ }^{11-13}$

\footnotetext{
'Department of Pathology, University Hospital of Alexandroupolis, Democritus University of Thrace, Alexandroupolis, Greece; ${ }^{2}$ Cancer Research UK, Molecular Oncology Laboratories, Weatherall Institute of Molecular Medicine, University of Oxford, Oxford, UK; ${ }^{3}$ Nuffield Division of Clinical Laboratory Sciences, Radcliffe Department of Medicine, John Radcliffe Hospital, Oxford OX3 9DU, UK; ${ }^{4} 1$ st Cardiac Surgery Department, Onassis Cardiac Surgery Center, 17674 Athens, Greece and ${ }^{5}$ Department of Radiotherapy/ Oncology, University Hospital of Alexandroupolis, Democritus University of Thrace, Alexandroupolis, Greece Correspondence: Michael I. Koukourakis (targ@her.forthnet.gr)
}

Received: 13 December 2019 Revised: 13 January 2020 Accepted: 30 January 2020 Published online: 18 February 2020 
In a previous study, we showed that CA9 is strongly expressed in about a third of NSCLC, and is correlated with angiogenic pathways and poor prognosis in operable disease. ${ }^{14}$ In this study, we provide evidence that CA9 is also involved in intratumoural immunosuppression pathways.

\section{METHODS}

A series of 98 tissue samples of primary non-small-cell lung carcinomas (NSCLC), from patients treated with surgery, were retrieved from the archives of Pathology, Democritus University of Thrace. Patient and disease characteristics are shown in Table 1.

Immunohistochemistry

Immunohistochemistry was performed on 3- $\mu$ m-thick formalinfixed paraffin-embedded tissue sections. Details in the methodology applied have been previously reported..$^{15}$ The Dako EnVision FLEX kit was used. The well-validated rabbit monoclonal PD-L1 antibody (clone CAL10, Biocare Medical, CA, USA) was used at a dilution of 1:100 and 60-min incubation at room temperature. ${ }^{16}$ For $\mathrm{FOXP3}^{+}$Treg detection, we used 'in house' undiluted hybridoma supernatant from the well-validated murine monoclonal antibody 236A/E7 that is widely used for FOXP3 detection in routinely fixed tissues. ${ }^{17}$ For CA9 detection, the mouse monoclonal M75 antibody was used, at dilution 1/200 and 60min incubation.

In each run of immunohistochemical staining, a tissue section from normal gastric or gallbladder tissue (Supplementary Fig. 1s) was included as a CA9-positive control. Normal lung tissue was used as a negative control. Tissue sections from a reactive (inflammatory) lymph node with extensive presence of FOXP3+ lymphocytes were used as a positive control in each immunohistochemistry run. FOXP3-positive, when present, and negative lymphocytes function also as a positive or negative internal control, respectively, in each slide.

The percentage of cancer cells with membrane CA9 and with strong membrane (with or without cytoplasmic) PD-L1 reactivity was assessed in all available optical fields at $\times 200$ magnification, and the mean values were used to score each case. Cases were grouped as negative (lack of expression), as of low (limited expression in $1-9 \%$ of cells), medium (10-49\% of cells) and high expression ( $>50$ of cells).

Assessment of TIL score and FOXP3+ TIL (FIL) score

Tumour-infiltrating lymphocytes (TILs) were assessed in the FOXP3-immunostained slides. The number of haematoxylinstained TILs was assessed in the stroma (not in tumour nests), in the entire tissue section and in $\times 40$ optical fields, and the mean value defined the final score for each case. Lymphocytes were recognised by morphological criteria, and there was no T-cell-specific staining applied. All mononuclear cells (including lymphocytes and plasma cells) were taken into account, while polymorphonuclear leukocytes and macrophages are excluded. Four different TIL-score categories were initially defined subjectively for scoring (minimal, low, medium and high). Counting the absolute numbers of lymphocytes per optical field, it was noted that TIL-score 1 (or minimal) defined cases with 1-10 lymphocytes/o.f., 2 (or low) 10-70 lymphocytes/o.f., 3 (or medium) 70-150 lymphocytes/o.f. and 4 (or high) $>150$ lymphocytes/o.f.

The percentage of FOXP3-expressing lymphocytes among the TILs present in the tumour stroma was assessed in the entire tissue section, in $\times 40$ optical fields, and the mean score was used for each case. This score provides only the \% of TILs expressing FOXP3, and does not reveal the extent of FOXP3 lymphocytic infiltration in the tissue, which also depends upon the extent of TIL presence. The FIL score was therefore assessed as the product of 'TIL-score' $\times$ '\% FOXP3+ TILs'.
Table 1. Patients' characteristics $(n=98)$.

\begin{tabular}{|c|c|}
\hline \multicolumn{2}{|l|}{ Age } \\
\hline Median & 68 \\
\hline Range & $32-81$ \\
\hline \multicolumn{2}{|l|}{ Sex } \\
\hline Male & 86 \\
\hline Female & 12 \\
\hline \multicolumn{2}{|l|}{ Stage } \\
\hline 1 & 46 \\
\hline II & 22 \\
\hline III & 30 \\
\hline IV & 0 \\
\hline \multicolumn{2}{|l|}{ Tumour cell type } \\
\hline Squamous cell carcinoma & 58 \\
\hline Adenocarcinoma & 22 \\
\hline Large-cell carcinoma & 18 \\
\hline \multicolumn{2}{|l|}{ Histology grade } \\
\hline \multicolumn{2}{|l|}{ Squamous cell carcinoma } \\
\hline 1 & 45 \\
\hline 2 & 8 \\
\hline 3 & 5 \\
\hline \multicolumn{2}{|l|}{ Adenocarcinoma } \\
\hline 1 & 7 \\
\hline 2 & 12 \\
\hline 3 & 3 \\
\hline \multicolumn{2}{|l|}{ Follow-up in months } \\
\hline Median & 46 \\
\hline Range & $26-112$ \\
\hline
\end{tabular}

Statistical analysis

Statistical analysis was performed using the GraphPad Prism 5.0 package. The chi-square or Fisher's exact $t$ test was used to compare categorical variables as appropriate. Kaplan-Meier survival curves were used to assess the impact of assessed variables on the diseasespecific overall survival of patients. A Cox's proportional hazard regression model using backward elimination was applied to assess the effect of the parameters on the death events. These included the CA9 expression (low/negative vs. medium/high), stage (1 vs. 2 vs. 3), the TIL score $(1,2$ vs. 3,4$)$ and the FIL score ( 0 vs. positive). A $p$-value of $<0.05$ was used for significance.

\section{RESULTS}

\section{Expression of CA9}

Membrane expression of CA9 was noted in a varying percentage of cancer cells, ranging from 0 to $90 \%$ (median 5\%, mean $16.7 \%$ ). No expression (0\%) was noted in $33 / 98$, low expression (1-9\%) in $21 / 33$, medium expression (10-49\%) in 32/98 and high $(>50)$ in $12 / 98$ cases. Figure $1 a-d$ shows representative immunohistochemical images from these four staining patterns.

There was no association between stage, histology, tumour grade and CA9 expression. Regarding the association of CA9 with PD-L1 expression, there was no significant association, as 14/44 (31.8\%) cases with medium/high CA9 expression had medium/ high PD-L1 expression vs. 9/54 (18.5\%) of cases with negative/low CA9 expression $(p=0.09)$.

Lymphocytic infiltration

Out of 98 cases, $20(20.5 \%)$ had a TIL score equal to 1, $36(36.7 \%)$ a score of $2,26(26.5 \%)$ a score of 3 and $16(16.3 \%)$ a score 

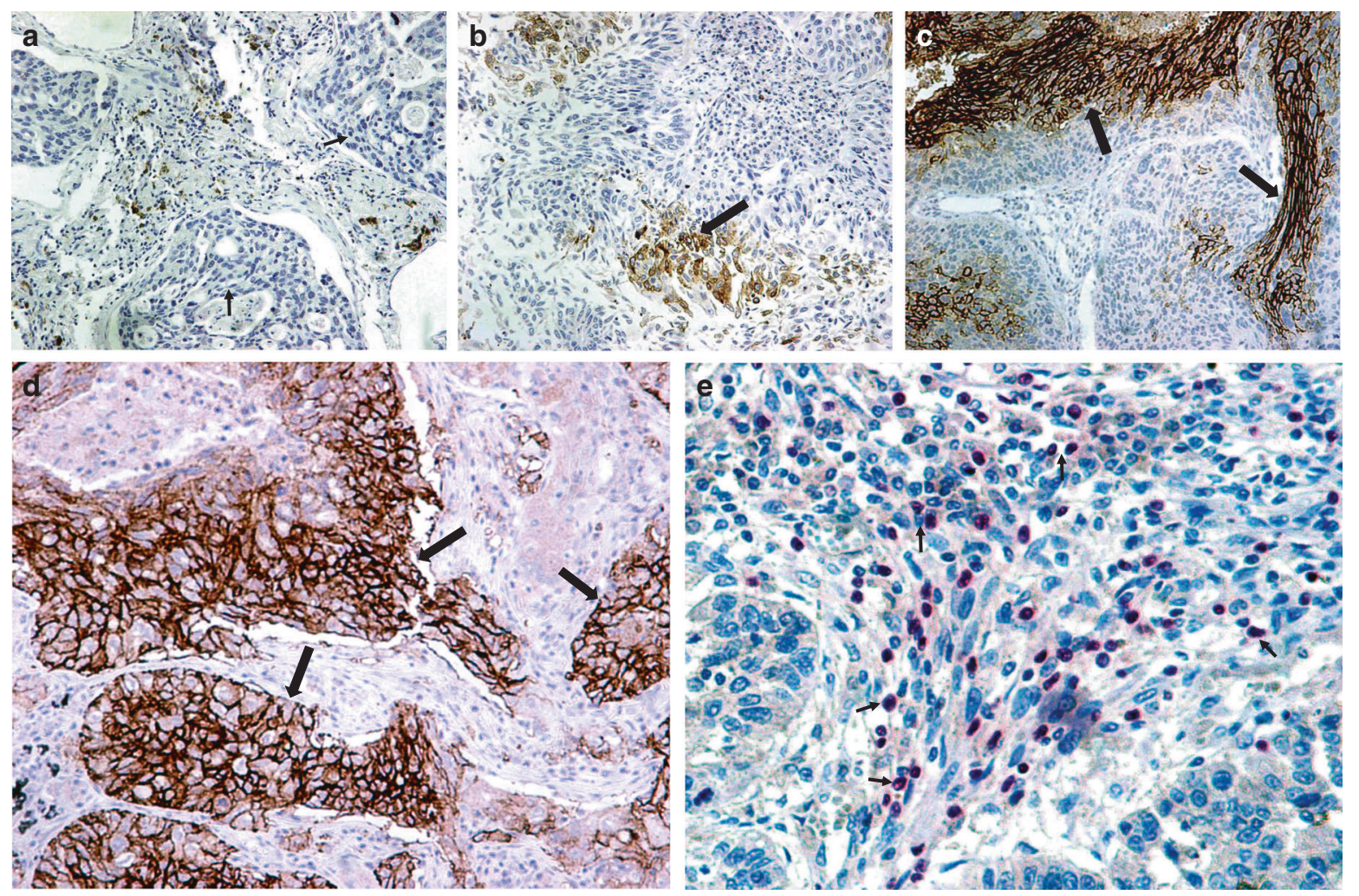

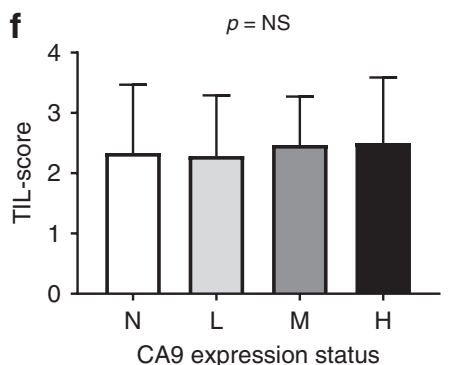

No pts $\begin{array}{llll}33 & 21 & 32 & 12\end{array}$

2

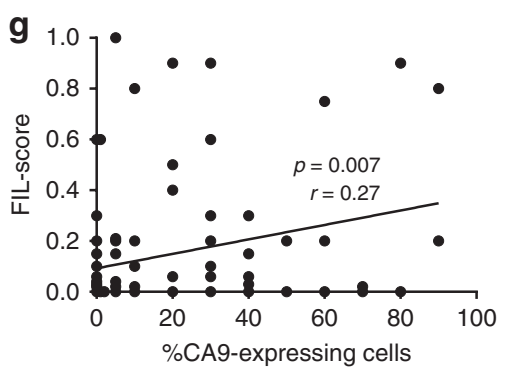

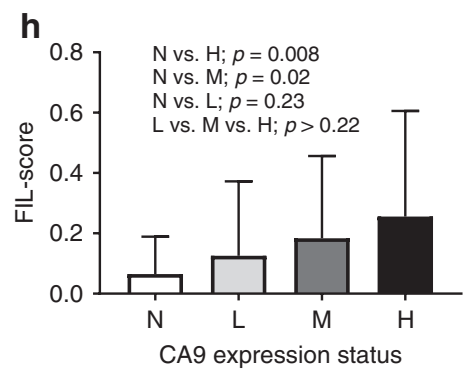

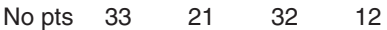

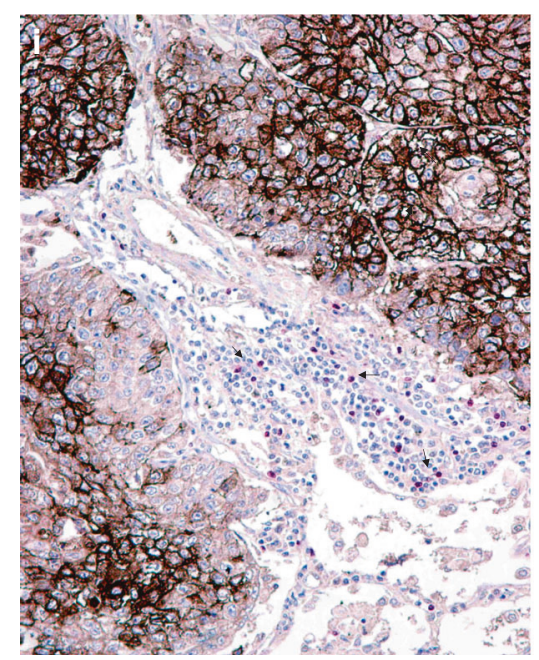

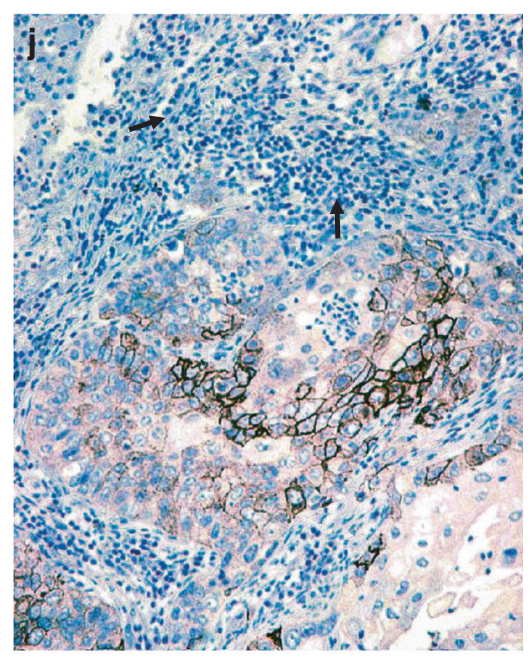

k

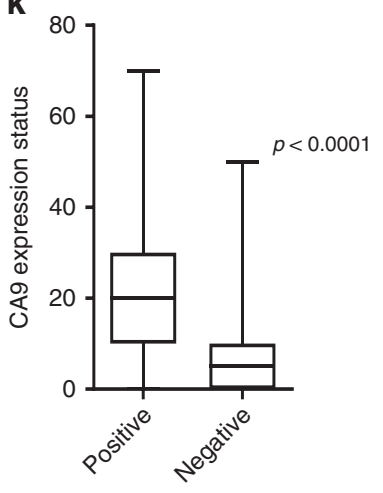

$\%$ FOXP3 + lymphocytes of 4 . The percentage of TILs with FOXP3 expression ranged from 0 to $50 \%$ (median 1\%, mean $6 \%$ and 75 th percentile $10 \%)$. Figure 1e shows a case with extensive presence of FOXP3+lymphocytes in the tumour stroma. The FIL score
('TIL-score' $\times$ '\%FOXP3+cells') ranged from 0 to 1 (median 0.02). Out of 98 cases, $47(47.9 \%)$ had a FIL score equal to 0 (zero '0'), $35(35.7 \%)$ had a score of $0.02-0.2$ and $16(16.3 \%)$ a score of $0.21-1$. 
Fig. 1 Immunohistochemical figures and figures showin associations between CA9 expression and lymphocyte-related parameters. a Immunohistochemical image showing lack (grouped as negative) of cancer cell CA9 reactivity (arrows) in a squamous cell lung cancer (magnification $\times 20$ ). b Immunohistochemical image showing sporadic (grouped as low) staining (arrows) of cancer cell CA9 reactivity in a squamous cell lung cancer (magnification $\times 20$ ). c Immunohistochemical image showing CA9 staining (arrows) in less than $50 \%$ of cancer cells (grouped as medium) in a squamous cell lung cancer (magnification $\times 20$ ). $\mathbf{d}$ Immunohistochemical image showing extensive membrane CA9 reactivity (arrows) in $>50 \%$ of cancer cells of a squamous cell lung cancer (magnification $\times 20$ ). e Immunohistochemical image showing intense infiltration of tumour stroma by FOXP3 + lymphocytes (arrows), in a squamous cell lung cancer (magnification $\times 40$ ). $\mathbf{f}$ TIL score according to the four CA9 expression categories (absence/N vs. low/L vs. medium/M vs. high/H expression). Bars show standard deviation. $\mathbf{g}$ Linear regression analysis of the percentage of CA9-expressing cancer cells and the FIL score. $\mathbf{h}$ FIL score according to the four CA9 expression categories (absence/N vs. low/L vs. medium/M vs. high/H expression). Bars show standard deviation. i Double immunostaining for CA9 and FOXP3 in a squamous cell lung cancer extensively expressing CA9; arrows show FOXP3+lymphocytes (magnification $\times 20$ ). $\mathbf{j}$ Double immunostaining for CA9 and FOXP3+ in a squamous cell lung cancer showing poor expression of CA9 in cancer cells, and lack of expression of FOXP3 by tumour-infiltrating lymphocytes (arrows) (magnification $\times 20$ ). k Percentage of FOXP3+ infiltrating lymphocytes in the stroma according to the extent of expression of CA9 adjacent to the stroma cancer cells, assessed in 20 selected double CA9/FOXP3-immunostained tissue slides. Bars show standard deviation (magnification $\times 20$ ).

Correlation of CA9 with lymphocyte parameters

There was no significant difference between the TIL scores obtained in the four groups defined by CA9 expression levels (Fig. 1f). Linear regression analysis revealed a significant direct association between CA9 expression and FIL score $(p=0.007$, $r=0.27$, Fig. 1g). High CA9 expression had a mean FIL score of 0.25 vs. 0.06 in tumours lacking CA9 expression $(p=0.008$, Fig. 1h).

We further performed double staining for CA9 and FOXP3 on 20 selected cases that expressed CA9 in $30-60 \%$ of the cancer cell population, containing areas with positive expression and lack of expression. In these slides, we assessed the percentage of FOXP3 + lymphocytes in the stroma adjacent to CA9-positive and negative areas (a total of paired 60 areas were evaluated). This was significantly higher in the stroma-adjacent cancer cell areas with strong CA9 expression (median value $20 \%$ vs. $5 \%$, mean value $22.3 \%$ vs. $9.2 \%, p<0.00001$ ) (Fig. $1 \mathrm{i}-\mathrm{k}$ ).

Survival analysis

The absence of, or low, CA9 expression defined favourable survival curves compared with medium-/high-expression groups, but the difference did not reach statistical significance (Fig. 2a, $p=0.15$ ). Intense infiltration of the tumour stroma by TILs (TIL score 3,4$)$ was significantly related to a better prognosis (Fig. 2b, $p=0.05$ ). Double stratification, according to TIL score and CA9 expression, showed that patients with medium/high CA9 expression and low TIL score $(1,2)$ had significantly poorer survival compared with all other groups (Fig. 2c, $p<0.04$ ).

For categorical analysis, the FIL score was divided into two categories ( 0 vs. $>0$ ), thus zero vs. positive. Overall, there was no association of FIL score with overall survival $(p=0.91)$. Doublestratification analysis according to CA9 expression (negative/low vs. medium/high) and FIL score (zero vs. positive) did not show groups of patients with different prognosis (Fig. 3a). Despite the low number of cases, a similar double-stratification analysis in stage III patients showed that patients who lacked CA9 expression and FOXP3+lymphocytes had a better prognosis, although of marginal significance ( $p=0.07$, Fig. $3 b$ ).

In a multivariate analysis, including stage, histology, TIL score, FIL score and CA9, stage ( $p<0.0001, \mathrm{HR} 1.95,95 \% \mathrm{Cl}: 1.3-2.7)$ and TIL score $(p=0.05$, HR $0.55,95 \% \mathrm{Cl}: 0.2-1.0)$ were independent prognostic variables of death events.

\section{DISCUSSION}

Cytotoxic T-cell activity, mainly mediated by activated CD8+ T cells (Tcyt), is important in the maintenance of an immunological equilibrium that impedes tumour growth. ${ }^{18}$ Shifting this equilibrium towards tumour elimination, by targeting immune coinhibitory molecules that suppress Tcyt activity, is under thorough clinical and laboratory investigation. Nevertheless, Tcyt activity is also regulated by a subset of $\mathrm{CD} 4+/ \mathrm{CD} 25+$ regulatory $\mathrm{T}$ cells
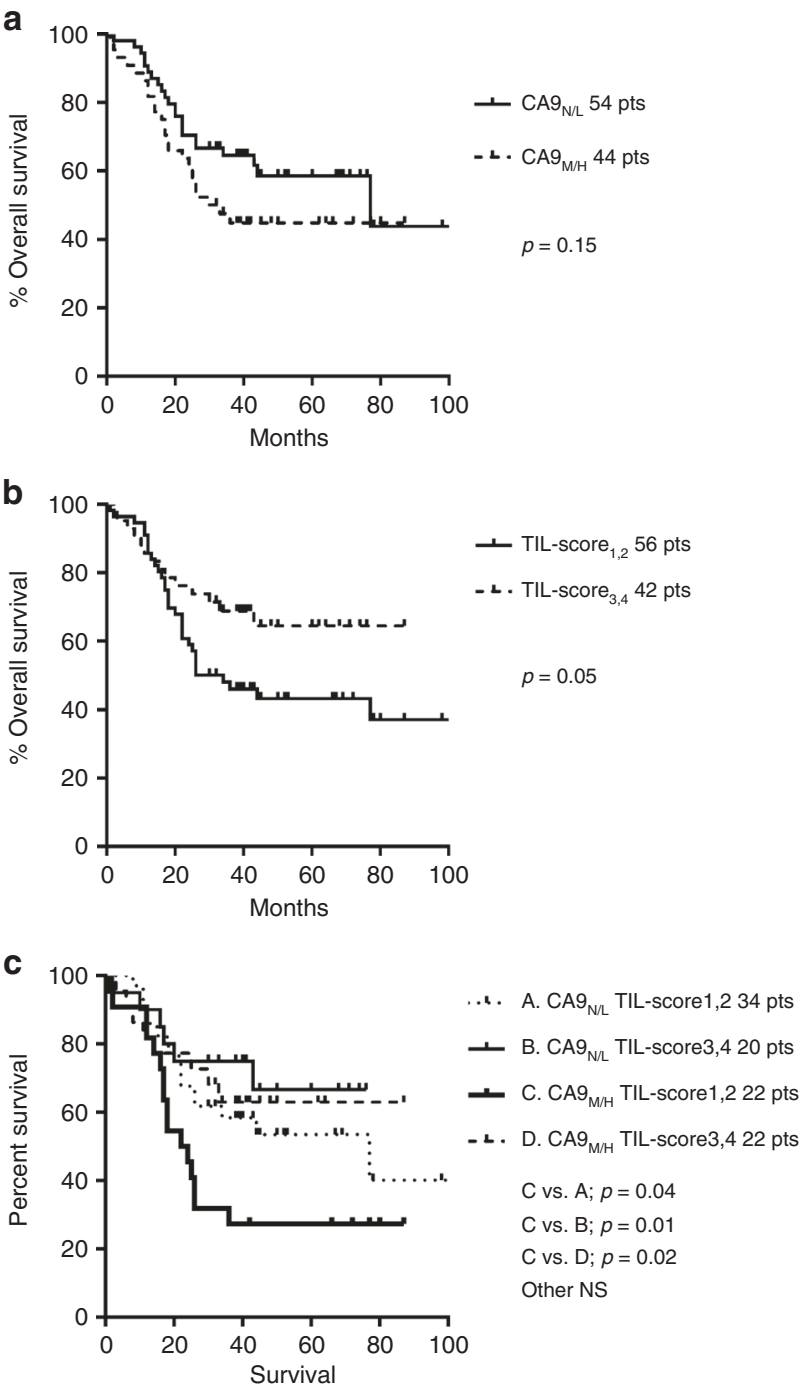

Fig. 2 Kaplan-Meier overall (disease-specific) survival curves. a Stratified for CA9 expression (negative-N/low-L vs. medium$\mathrm{M} /$ high-H), b stratified for TIL score (low 1,2 vs. high 3,4) and c double stratification for CA9 and TIL score.

that express FOXP3 (regulatory T cells or Tregs). FOXP3 is essential for the development of natural $\mathrm{CD} 4^{+/} \mathrm{CD} 25^{+}$Tregs in humans, ${ }^{19}$ although it also characterises CD4 + Tregs independently of CD25 expression. ${ }^{20}$ Under normal conditions, circulating Tregs represent $5 \%$ of the total CD4+lymphocytic population, while their presence increases in cancer patients. ${ }^{21}$ 

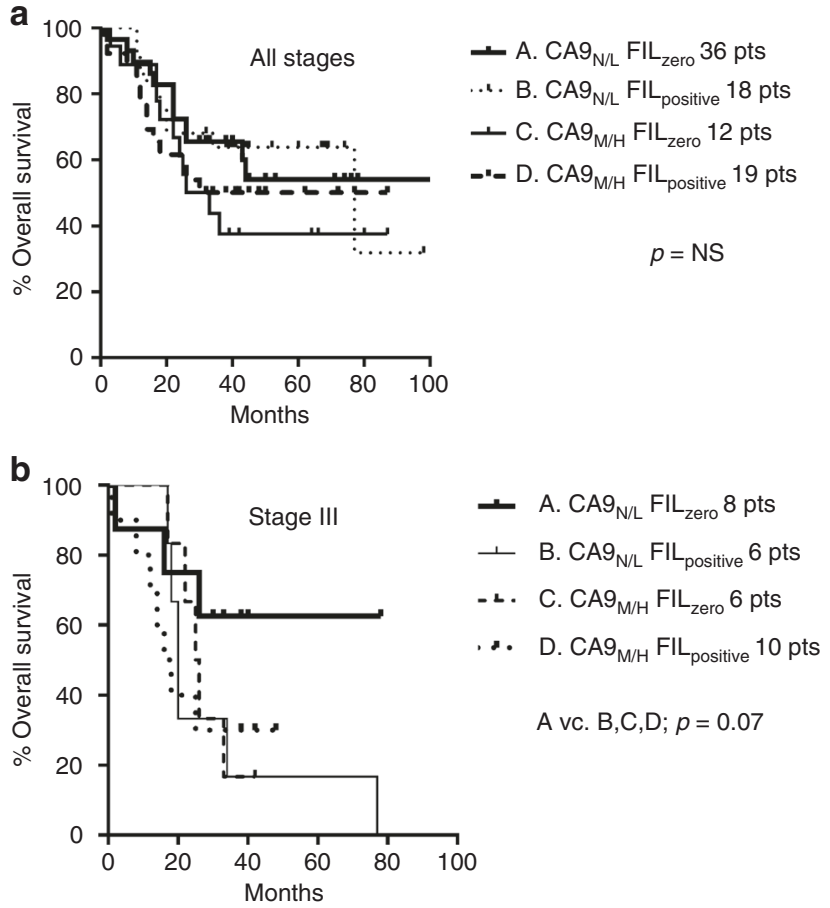

Fig. 3 Figures of survival analysis. Kaplan-Meier overall (diseasespecific) survival curves stratified for CA9 expression (negative- $\mathrm{N}$ / low $-\mathrm{L}$ vs. medium $-\mathrm{M} / \mathrm{high}-\mathrm{H}$ ) and FIL score (zero vs. positive) in all cases (a) and in stage III cases (b).

Intense FOXP3 + lymphocyte infiltration relates to poor prognosis, ${ }^{22}$ and indeed this has been documented in patients with NSCLC. ${ }^{23-28}$ In a recent study, we found that tumour infiltration by FOXP3 + Tregs occurs early in the development of NSCLC, and adversely affects the post-operative outcome. ${ }^{15}$ Tregs may compromise the activity of immune-checkpoint inhibitors, so that assessment of their presence could be a useful marker to guide immunotherapy. Therapeutic interventions targeting this very T-cell population may also prove to be important to improve survival of patients. It seems, therefore, important to study the reasons for Treg expansion and tumour infiltration in cancer patients, aiming to devise methods to remove this principal obstacle, and enhance the activity of modern immunotherapy.

A hypoxic tumour microenvironment promotes local immunosuppression through many biological pathways, including lactate release by cancer cells, acidosis, production of immunosuppressive molecules like adenosine and HIF1a-driven overexpression of checkpoint co-inhibitory molecules, like PD-L1 and CD47., 5,6,29 HIF1a is a transcriptional regulator of CA9 and of lactate dehydrogenase $A .^{30,31}$ In a previous study, we showed that LDHA is directly linked with PD-L1 expression in lung cancer cells, a relation that can be explained by the hypoxic environment of LDHA-expressing tumours. ${ }^{32}$ In this study, we also noted a trend for CA9-overexpressing tumours to have higher levels of PD-L1 expression. These data confirm at the clinical level that a hypoxic tumour microenvironment promotes PD-L1 expression and immune-checkpoint inhibition.

Aside to this direct effect of hypoxia on checkpoint inhibitory molecule expression, several studies suggest that cytotoxic T-cell proliferation and activity are severely compromised under the acidic conditions of the tumour microenvironment. ${ }^{10,11}$ In addition, chemokines produced by cancer cells under acidic and hypoxic conditions, induce chemotaxis of Tregs. ${ }^{33}$ HIF activation also seems to induce differentiation of CD4+ cells to FOXP3- expressing Tregs, either by direct binding to the FOXP3 regulatory region or by induction of TGF $\beta^{34,35}$

Carbonic anhydrase 9 is a HIF1a-regulated downstream gene, and is involved in the acidification of the extracellular matrix by hydrating carbon dioxide to produce bicarbonate and protons. ${ }^{9}$ In this study, we provide evidence that CA9 expression by cancer cells is directly related to intense infiltration of the tumour stroma by FOXP3+ Tregs in NSCLC. A similar finding has been reported by Yan et al. ${ }^{36}$ in breast cancer, who also reported that hypoxia upregulated CXCR4 on Tregs. This association can be explained by the fact that CA9 is a hypoxia-regulated gene, directly regulated by HIF1a, so that the correlation found between CA9 expression and FOXP3 + TILs may be an indirect result of the hypoxic tumour environment and not a direct effect of acidity conferred by the carbonic acid released. Whether acidity per se can directly promote Treg chemotaxis, or can, similarly to hypoxia, promote differentiation of CD4+ T cells to FOXP3+ Tregs, remains obscure.

In any case, CA9, appearing to be a marker of tumour aggressiveness by reflecting active hypoxia pathways, is also a marker of local immunosuppression and Treg accumulation. Indeed, tumours with low CA9 expression and intense TIL presence in the tumour stroma defined a group of patients with optimal prognosis. Of interest, and despite the low number of patients analysed with stage III disease, lack of CA9 expression and absence of FOXP3+ lymphocytic infiltration also defined a group with better prognosis.

It is concluded that CA9 is a marker strongly associated with FOXP3 + regulatory T-cell abundance in the tumour environment of NSCLC. Whether CA9-positive NSCLCs are resistant to immunecheckpoint inhibitors should be sought in translational studies. Such patients would, eventually, benefit from a combination of immunotherapy with pharmaceutical agents that neutralise extracellular tumour acidity, such as CA9 and bicarbonate transport inhibitors. ${ }^{37-40}$

\section{ACKNOWLEDGEMENTS}

We would like to thank Ms K. Devetzi for assisting in the immunohistochemical elaboration of the tissue material.

\section{AUTHOR CONTRIBUTIONS}

A.G., A.L.H. and M.I.K.: conception and design, analysis and interpretation of the data and writing of the paper. A.H.B.: analysis and interpretation of the data, writing of the paper. C.A.C.: acquisition of the data, analysis and interpretation of the data and writing of the paper.

\section{ADDITIONAL INFORMATION}

Ethics approval and consent to participate Ethical approval was obtained from the Scientific Committee and the Ethics Research Committee of the University Hospital of Alexandroupolis (study approval number ES11-26-11-18). As the study is based on archival material, and anonymity of patients was guaranteed, patient informed consent was waved by the ethics board. The study was conducted according to the criteria set by the declaration of Helsinki.

Consent to publish There are no individual person's data included in the paper.

Data availability All data reported in the study are available in our departments.

Competing interests A.L.H. is the Editor-in-Chief of the British Journal of Cancer. The remaining authors declare no competing interests.

Funding information The study has been financially supported by the Democritus University of Thrace Special Account, project no 81006.

Supplementary information is available for this paper at https://doi.org/10.1038/ s41416-020-0756-3. 
Note This work is published under the standard license to publish agreement. After 12 months the work will become freely available and the license terms will switch to a Creative Commons Attribution 4.0 International (CC BY 4.0).

Publisher's note Springer Nature remains neutral with regard to jurisdictional claims in published maps and institutional affiliations.

\section{REFERENCES}

1. Doroshow, D. B., Sanmamed, M. F., Hastings, K., Politi, K., Rimm, D. L., Chen, L. et al. Immunotherapy in non-small cell lung cancer, facts and hopes. Clin. Cancer Res. 25, 4592-4602 (2019).

2. Garon, E. B., Rizvi, N. A., Hui, R., Leighl, N., Balmanoukian, A. S., Eder, J. P. et al. KEYNOTE-001 Investigators. Pembrolizumab for the treatment of non-small-cell lung cancer. N. Engl. J. Med. 372, 2018-2028 (2015).

3. Ribas, A. \& Wolchok, J. D. Cancer immunotherapy using checkpoint blockade. Science 359, 1350-1355 (2018).

4. Corzo, C. A., Condamine, T., Lu, L., Cotter, M. J., Youn, J. I., Cheng, P. et al. HIF-1a regulates function and differentiation of myeloid-derived suppressor cells in the tumor microenvironment. J. Exp. Med. 207, 2439-2453 (2010).

5. Zhang, H., Lu, H., Xiang, L., Bullen, J. W., Zhang, C., Samanta, D. et al. HIF-1 regulates CD47 expression in breast cancer cells to promote evasion of phagocytosis and maintenance of cancer stem cells. Proc. Natl Acad. Sci. USA 112, E6215-E6223 (2015).

6. Noman, M. Z., Desantis, G., Janji, B., Hasmim, M., Karray, S., Dessen, P. et al. PD-L1 is a novel direct target of HIF-1a, and its blockade under hypoxia enhanced MDSC-mediated T cell activation. J. Exp. Med. 211, 781-790 (2014).

7. Damaghi, M., Wojtkowiak, J. W. \& Gillies, R. J. pH sensing and regulation in cancer. Front. Physiol. 4, 370 (2013).

8. Fan, J., Kamphorst, J. J., Mathew, R., Chung, M. K., White, E., Shlomi, T. et al. Glutamine-driven oxidative phosphorylation is a major ATP source in transformed mammalian cells in both normoxia and hypoxia. Mol. Syst. Biol. 9, 712 (2013).

9. Sedlakova, O., Svastova, E., Takacova, M., Kopacek, J., Pastorek, J. \& Pastorekova, S. Carbonic anhydrase IX, a hypoxia-induced catalytic component of the $\mathrm{pH}$ regulating machinery in tumors. Front. Physiol. 4, 400 (2014).

10. Gallagher, F. A., Sladen, H., Kettunen, M. I., Serrao, E. M., Rodrigues, T. B., Wright, A. et al. Carbonic anhydrase activity monitored in vivo by hyperpolarized $13 \mathrm{C}$ magnetic resonance spectroscopy demonstrates its importance for $\mathrm{pH}$ regulation in tumors. Cancer Res. 75, 4109-4118 (2015).

11. Nakagawa, Y., Negishi, Y., Shimizu, M., Takahashi, M., Ichikawa, M. \& Takahashi, $\mathrm{H}$. Effects of extracellular $\mathrm{pH}$ and hypoxia on the function and development of antigen-specific cytotoxic T lymphocytes. Immunol. Lett. 167, 72-86 (2015).

12. Calcinotto, A., Filipazzi, P., Grioni, M., lero, M., De Milito, A., Ricupito, A. et al. Modulation of microenvironment acidity reverses anergy in human and murine tumor-infiltrating T lymphocytes. Cancer Res. 72, 2746-2756 (2012).

13. Fischer, B., Müller, B., Fisch, P. \& Kreutz, W. An acidic microenvironment inhibits antitumoral non-major histocompatibility complex-restricted cytotoxicity, implications for cancer immunotherapy. J. Immunother. 23, 196-207 (2000).

14. Giatromanolaki, A., Koukourakis, M. I., Sivridis, E., Pastorek, J., Wykoff, C. C., Gatter, K. C. et al. Expression of hypoxia-inducible carbonic anhydrase-9 relates to angiogenic pathways and independently to poor outcome in non-small cell lung cancer. Cancer Res. 61, 7992-7998 (2001).

15. Giatromanolaki, A., Banham, A. H., Harris, A. L. \& Koukourakis, M. I. FOXP3 infiltrating lymphocyte density and PD-L1 expression in operable non-small cell lung carcinoma. Exp. Lung Res. 45, 76-83 (2019).

16. Teixidó, C., Vilariño, N., Reyes, R. \& Reguart, N. PD-L1 expression testing in nonsmall cell lung cancer. Ther. Adv. Med. Oncol. 10, 1758835918763493 (2018).

17. Bates, G. J., Fox, S. B., Han, C., Leek, R. D., Garcia, J. F., Harris, A. L. et al. Quantification of regulatory $T$ cells enables the identification of high-risk breast cancer patients and those at risk of late relapse. J. Clin. Oncol. 24, 5373-5380 (2006).

18. Dunn, G. P., Bruce, A. T., Ikeda, H., Old, L. J. \& Schreiber, R. D. Cancer immunoediting, from immunosurveillance to tumor escape. Nat. Immunol. 3, 991-998 (2002).

19. Hori, S., Nomura, T. \& Sakaguchi, S. Control of regulatory T cell development by the transcription factor Foxp3. Science 299, 1057-1061 (2003).
20. Fontenot, J. D., Rasmussen, J. P., Williams, L. M., Dooley, J. L., Farr, A. G. \& Rudensky, A. Y. Regulatory $T$ cell lineage specification by the forkhead transcription factor foxp3. Immunity 22, 329-341 (2005).

21. Phillips, J. D., Knab, L. M., Blatner, N. R., Haghi, L., DeCamp, M. M., Meyerson, S. L. et al. Preferential expansion of pro-inflammatory Tregs in human non-small cell lung cancer. Cancer Immunol. Immunother. 64, 1185-1191 (2015).

22. Bignone, P. A. \& Banham, A. H. FOXP3+ regulatory T cells as biomarkers in human malignancies. Expert Opin. Biol. Ther. 8, 1897-1920 (2008).

23. Usó, M., Jantus-Lewintre, E., Bremnes, R. M., Calabuig, S., Blasco, A., Pastor, E. et al. Analysis of the immune microenvironment in resected non-small cell lung cancer, the prognostic value of different T lymphocyte markers. Oncotarget 7 , 52849-52861 (2016).

24. Kadota, K., Nitadori, J. I. \& Adusumilli, P. S. Prognostic value of the immune microenvironment in lung adenocarcinoma. Oncoimmunology 2, e24036 (2013).

25. Zhang, G. Q., Han, F., Fang, X. Z. \& Ma, X. M. CD4+, IL17 and Foxp3 expression in different pTNM stages of operable non-small cell lung cancer and effects on disease prognosis. Asian Pac. J. Cancer Prev. 13, 3955-3960 (2012).

26. Shimizu, K., Nakata, M., Hirami, Y., Yukawa, T., Maeda, A. \& Tanemoto, K. Tumorinfiltrating Foxp3+ regulatory $\mathrm{T}$ cells are correlated with cyclooxygenase-2 expression and are associated with recurrence in resected non-small cell lung cancer. J. Thorac. Oncol. 5, 585-590 (2010).

27. Suzuki, K., Kadota, K., Sima, C. S., Nitadori, J., Rusch, V. W., Travis, W. D. et al. Clinical impact of immune microenvironment in stage I lung adenocarcinoma, tumor interleukin-12 receptor $\beta 2$ (IL-12Rß2), IL-7R, and stromal FoxP3/CD3 ratio are independent predictors of recurrence. J. Clin. Oncol. 31, 490-498 (2013).

28. Tao, H., Mimura, Y., Aoe, K., Kobayashi, S., Yamamoto, H., Matsuda, E. et al. Prognostic potential of FOXP3 expression in non-small cell lung cancer cells combined with tumor-infiltrating regulatory T cells. Lung Cancer 75, 95-101 (2012).

29. Li, Y., Patel, S. P., Roszik, J. \& Qin, Y. Hypoxia-driven immunosuppressive metabolites in the tumor microenvironment, new approaches for combinational immunotherapy. Front. Immunol. 9, 1591 (2018).

30. Wykoff, C. C., Beasley, N. J., Watson, P. H., Turner, K. J., Pastorek, J., Sibtain, A. et al. Hypoxia-inducible expression of tumor-associated carbonic anhydrases. Cancer Res. 60, 7075-7083 (2000).

31. Semenza, G. L., Jiang, B. H., Leung, S. W., Passantino, R., Concordet, J. P., Maire, P. et al. Hypoxia response elements in the aldolase $A$, enolase 1 , and lactate dehydrogenase A gene promoters contain essential binding sites for hypoxiainducible factor 1. J. Biol. Chem. 271, 32529-32537 (1996).

32. Giatromanolaki, A., Koukourakis, I. M., Balaska, K., Mitrakas, A. G., Harris, A. L., Koukourakis, M. I. Programmed death-1 receptor (PD-1) and PD-ligand-1 (PD-L1) expression in non-small cell lung cancer and the immune-suppressive effect of anaerobic glycolysis. Med. Oncol. 36, 76 (2019).

33. Facciabene, A., Peng, X., Hagemann, I. S., Balint, K., Barchetti, A., Wang, L. P. et al. Tumour hypoxia promotes tolerance and angiogenesis via CCL28 and T(reg)cells. Nature 475, 226-230 (2011).

34. Clambey, E. T., McNamee, E. N., Westrich, J. A., Glover, L. E., Campbell, E. L., Jedlicka, P. et al. Hypoxia-inducible factor-1 alpha-dependent induction of FoxP3 drives regulatory T-cell abundance and function during inflammatory hypoxia of the mucosa. Proc. Natl Acad. Sci. USA 109, E2784-E2793 (2012).

35. Deng, B., Zhu, J. M., Wang, Y., Liu, T. T., Ding, Y. B., Xiao, W. M. et al. Intratumor hypoxia promotes immune tolerance by inducing regulatory T cells via TGF- $\beta 1$ in gastric cancer. PLoS ONE 8, e63777 (2013).

36. Yan, M., Jene, N., Byrne, D., Millar, E. K., O’Toole, S. A., McNeil, C. M. et al. Recruitment of regulatory $\mathrm{T}$ cells is correlated with hypoxia-induced CXCR4 expression, and is associated with poor prognosis in basal-like breast cancers. Breast Cancer Res. 13, R47 (2011).

37. Pilon-Thomas, S., Kodumudi, K. N., El-Kenawi, A. E., Russell, S., Weber, A. M., Luddy, K. et al. Neutralization of tumor acidity improves antitumor responses to immunotherapy. Cancer Res. 76, 1381-1390 (2016).

38. Scurr, M., Pembroke, T., Bloom, A., Roberts, D., Thomson, A., Smart, K. et al. Lowdose cyclophosphamide induces antitumor T-cell responses, which associate with survival in metastatic colorectal cancer. Clin. Cancer Res. 23, 6771-6780 (2017).

39. da Motta, L. L., Ledaki, I., Purshouse, K., Haider, S., De Bastiani, M. A., Baban, D. et al. The BET inhibitor JQ1 selectively impairs tumour response to hypoxia and downregulates CA9 and angiogenesis in triple negative breast cancer. Oncogene 36, 122-132 (2017).

40. McIntyre, A., Hulikova, A., Ledaki, I., Snell, C., Singleton, D., Steers, G. et al. Disrupting hypoxia-induced bicarbonate transport acidifies tumor cells and suppresses tumor growth. Cancer Res. 76, 3744-3755 (2016). 